\title{
Preoperative locoregional staging of gastric cancer: is there a place for magnetic resonance imaging? Prospective comparison with EUS and multidetector computed tomography
}

\author{
Francesco Giganti • Elena Orsenigo $\cdot$ Paolo Giorgio Arcidiacono • \\ Roberto Nicoletti - Luca Albarello - Alessandro Ambrosi - Annalaura Salerno • \\ Antonio Esposito - Maria Chiara Petrone - Damiano Chiari - Carlo Staudacher • \\ Alessandro Del Maschio $\cdot$ Francesco De Cobelli
}

Received: 8 October 2014/ Accepted: 8 January 2015/Published online: 23 January 2015

(c) The International Gastric Cancer Association and The Japanese Gastric Cancer Association 2015

\begin{abstract}
Background The aim of this study was to prospectively compare the diagnostic performance of magnetic resonance imaging (MRI), multidetector computed tomography (MDCT) and endoscopic ultrasonography (EUS) in the preoperative locoregional staging of gastric cancer.

Methods This study had Institutional Review Board approval, and informed consent was obtained from all patients. Fifty-two patients with biopsy-proven gastric cancer underwent preoperative 1.5-T MRI, 64-channel MDCT and EUS. All images were analysed blind, and the results were compared with histopathological findings according to the seventh edition of the TNM classification. After the population had been divided on the basis of the local invasion (T1-3 vs T4a-b) and nodal involvement (N0 vs $\mathrm{N}+$ ), sensitivity, specificity, positive and negative
\end{abstract}

F. Giganti $(\bowtie) \cdot$ R. Nicoletti - A. Salerno - A. Esposito

A. Del Maschio - F. De Cobelli

Department of Radiology and Centre for Experimental Imaging,

San Raffaele Scientific Institute, Vita-Salute San Raffaele

University, Via Olgettina 60, 20132 Milan, Italy

e-mail: giganti.fra@gmail.com

E. Orsenigo $\cdot$ D. Chiari - C. Staudacher

Department of Surgery, San Raffaele Scientific Institute,

Vita-Salute San Raffaele University, Milan, Italy

P. G. Arcidiacono - M. C. Petrone

Department of Gastroenterology and Gastrointestinal

Endoscopy, San Raffaele Scientific Institute, Vita-Salute San

Raffaele University, Milan, Italy

L. Albarello

Pathology Unit, San Raffaele Scientific Institute, Milan, Italy

A. Ambrosi

Vita-Salute San Raffaele University, Milan, Italy predictive value, and accuracy were calculated and diagnostic performance measures were assessed using the McNemar test.

Results For $\mathrm{T}$ staging, EUS showed higher sensitivity (94\%) than MDCT and MRI (65 and $76 \% ; p=0.02$ and $p=0.08)$. MDCT and MRI had significantly higher specificity (91 and $89 \%)$ than EUS $(60 \%)(p=0.0009$ and $p=0.003$ ). Adding MRI to MDCT or EUS did not result in significant differences for sensitivity. For $\mathrm{N}$ staging, EUS showed higher sensitivity (92\%) than MRI and MDCT (69 and $73 \% ; p=0.01$ and $p=0.02$ ). MDCT showed better specificity (81\%) than EUS and MRI (58 and $73 \% ; p=0.03$ and $p=0.15$ ).

Conclusions Our prospective study confirmed the leading role of EUS and MDCT in the staging of gastric cancer and did not prove, at present, the value of the clinical use of MRI.

Keywords Gastric cancer - Endoscopic ultrasonography · Multidetector computed tomography - Magnetic resonance imaging $\cdot$ TNM staging

\section{Introduction}

Gastric cancer is one of the commonest malignancies worldwide, and both the prognosis and survival rate are poor for advanced stages [1]. Currently, the surgical approach is the only curative treatment, but few patients are candidates for resection at the time of presentation [2].

Therefore, an accurate preoperative staging allows rational treatment selection. Strategies range from endoscopic mucosal resection to preoperative neoadjuvant therapy, which is strongly recommended for locally advanced cases (with serosal invasion and/or nodal 
involvement) [3, 4]. Endoscopic ultrasonography (EUS) and multidetector computed tomography (MDCT) are the commonest techniques in the staging of gastric cancer, despite conflicting results having been reported [5-7].

EUS is invasive but capable of detecting all the wall layers and is regarded as the modality of choice for local staging, with an accuracy ranging from 65 to $92.1 \%$ for $\mathrm{T}$ stage [8] and from 66 to $90 \%$ for $\mathrm{N}$ stage $[9,10]$. However, this technique has a restricted field of view for nodal involvement, is highly operator dependent and cannot detect distant metastases.

Conversely, MDCT is non-invasive and is able to assess the presence of distant metastases. Its accuracy has constantly improved, for detecting both the invasion of gastric wall, ranging from 69 to $89 \%$ [11-13], and nodal involvement, ranging from 69 to $92 \%[14,15]$. Nevertheless, MDCT involves the use of ionizing radiation and cannot adequately differentiate all the gastric wall layers.

Recent advances in magnetic resonance imaging (MRI) [e.g. breath-hold sequences and diffusion-weighted imaging (DWI)] have improved the value of MRI for abdominal imaging, including imaging of gastric cancer [16]; in particular, on DWI pathological tissue is characterized by higher signal intensity than normal structures [17]. MRI studies report an accuracy ranging from 73.5 to $87.5 \%$ for T stage $[18,19]$ and from 55.2 to $65 \%$ for $\mathrm{N}$ stage $[19,20]$.

As is widely recognized, this technique provides high soft tissue contrast but has long acquisition times; moreover, motion artefacts (peristalsis, cardiovascular pulsation) are some important limitations.

To our knowledge, the only study where the three modalities were compared on the same cohort of patients affected by gastric cancer is that of Heye et al. [21]. They concluded that ex vivo endoluminal MRI was able to achieve adequate staging results compared with EUS and MDCT, suggesting that further investigations were needed to show if the potential role of endoluminal MRI could be transferred into a clinical in vivo setting. One drawback of that study, as we perceive it, was the comparison of preoperative EUS and MDCT findings with ex vivo endoluminal MRI findings for gastric specimens after gastrectomy.

Hence, our purpose was to compare the in vivo diagnostic performance of MRI, MDCT and EUS in the preoperative locoregional staging of gastric cancer.

\section{Methods}

This is a prospective study following the Standards for Reporting of Diagnostic Accuracy (STARD) guidelines, in accordance with the World Medical Association Declaration of Helsinki and good clinical practice guidelines.
The study was sponsored by the Gastrointestinal Unit of our institute and was performed in the Radiology Department and Centre for Experimental Imaging of our hospital. The protocol was approved by our medical ethics committee, and all patients provided written informed consent. The pharmaceutical companies were not involved in the design of the study, in the analysis of data or in the preparation of the manuscript.

All the authors contributed substantially to the conception and design of the study and the first, second and last authors vouch for the completeness and accuracy of the data reported and the fidelity of the study to the protocol.

\section{Patients}

Between November 2009 and October 2013, 114 consecutive patients with evidence of gastric cancer were referred to our institution.

Inclusion criteria for this study were (a) biopsy-proven Siewert II-III or gastric cancer, (b) no contraindications to preoperative imaging, (c) visible tumour with all three imaging techniques, and (d) fitness for surgery. Exclusion criteria were (a) EUS, MRI and MDCT contraindications $(n=3)$, (b) stage IV disease (metastases/peritoneal seeding demonstrated by peritoneal washing or laparoscopic biopsy) on MRI, MDCT or EUS $(n=25)$, and (c) neoadjuvant therapy before surgery $(n=34)$. Thus, the final study population consisted of 52 patients ( 33 men; 19 women; mean age $68.5 \pm 1.35$ years; age range 43-85 years).

Before surgical intervention patients underwent (1) 1.5T MRI including DWI, (2) 64-slice MDCT and (3) EUS on different days and then were treated with radical surgery according to (a) clinical status, (b) EUS preoperative staging and (c) MDCT preoperative staging [5-7]. Imaging and histopathological evaluation were performed using the seventh edition of TNM classification [22].

In our centre we routinely perform neoadjuvant therapy for locally advanced/node-positive gastric cancer [3]; all locally advanced patients enrolled in this study did not receive chemotherapy for reasons of comorbidity, contraindications or lack of consent.

MRI protocol and analysis

MRI scans were performed with a 1.5-T MRI system (Achieva, Philips Medical Systems, Best, The Netherlands) using a five-channel phased-array cardiac coil positioned according to tumour location, with cardiac and respiratory triggering.

Visceral distension was obtained by oral administration of $500 \mathrm{~mL}$ of water and ferumoxsil (Lumirem ${ }^{\circledR}$; Guerbet, 
Roissy CdG Cedex, France) before imaging; in the absence of contraindications, an intramuscular injection of scopolamine butylbromide $\left(20 \mathrm{mg}\right.$, Buscopan ${ }^{\circledR}$, Boehringer Ingelheim, Ingelheim, Germany) was administered after patient positioning. In accordance with the literature [23], we performed a T2-weighted study, with and without fat suppression, followed by DWI $\left(b=0-600 \mathrm{~s} / \mathrm{mm}^{2}\right)$, and a dynamic T1-weighted study with fat suppression during intravenous injection of gadobutrol (Gadovist ${ }^{\circledR}, 1 \mathrm{mmol} /$ $\mathrm{mL}$; Bayer Schering Pharma, Berlin, Germany) at $0.1 \mathrm{~mL}$ per kilogram of body weight with an automatic injector (Spectris MR, Medrad Europe, Maastricht, The Netherlands) at a rate of $2 \mathrm{~mL} / \mathrm{s}$. The total imaging time was approximately $40 \mathrm{~min}$.

The parameters for the T1-weighted MRI images were as follows: the shortest repetition time (TR) and echo time (TE); flip angle $10^{\circ}$; slice thickness $25 \mathrm{~mm}$; field of view $365 \mathrm{~mm} \times 289 \mathrm{~mm}$; matrix size (reconstructed) 288; acquisition time $94 \mathrm{~s}$; number of slices 65 . The parameters for the T2-weighted MRI images were as follows: TR 2,400 ms; TE $80 \mathrm{~ms}$; flip angle $90^{\circ}$; slice thickness $4 \mathrm{~mm}$; field of view $300 \mathrm{~mm} \times 280 \mathrm{~mm}$; matrix size (reconstructed) 288; acquisition time $150 \mathrm{~s}$; number of slices 18 . The parameters for the DWI study were as follows: TR 1.00 (beats) ms; TE $58 \mathrm{~ms}$; flip angle $90^{\circ}$; slice thickness $4 \mathrm{~mm}$; field of view $365 \mathrm{~mm} \times 319 \mathrm{~mm}$; matrix size (reconstructed) 336; acquisition time $104 \mathrm{~s}$; number of slices $30 ; b=0-600 \mathrm{~s} / \mathrm{mm}^{2}$.

MRI scans were reviewed independently by two radiologists (F.D.C. and F.G.; 20 and 5 years of experience in abdominal imaging, respectively) who were privy only to tumour location, as small or superficial gastric cancers can be difficult to detect. In the case of discordance, the opinion of a third radiologist (A.E.; 8 years of experience in abdominal imaging) was requested.

The anatomical appearance of gastric wall layers and the depth of infiltration $(\mathrm{T})$ were evaluated in accordance with earlier studies [24, 25]: T1 (enhanced tumour that does not penetrate the submucosa); T2 (clear continuous low signal intensity band or enhanced cancerous portion in correspondence with the low signal intensity band of the muscularis propria); $\mathrm{T} 3$ (enhanced tumour reaching the subserosa with no signs of infiltration); T4a (interrupted low signal intensity band or enhanced cancerous portions penetrating the serosa); T4b (continuous extension of the cancerous portion to the adjacent organs). Since there is still no worldwide consensus regarding dimensional criteria for $\mathrm{N}$ staging, round lymph nodes with a short-axis diameter greater than $6 \mathrm{~mm}$ (which is a widely used dimensional criterion for perigastric lymph nodes [26]) and showing hyperintensity on DWI were considered pathological.
MDCT protocol and analysis

MDCT scans were performed with a 64-slice scanner (Brilliance 64-channel scanner, Philips Medical Systems, Best, The Netherlands). Before the examination all patients received $500 \mathrm{~mL}$ of water orally for adequate stomach distension and, if there were no contraindications, an intravenous injection of scopolamine butylbromide (20 mg, Buscopan ${ }^{\circledR}$, Boehringer Ingelheim, Ingelheim, Germany). A non-ionic contrast agent (iopromide; Ultravist $370^{\circledR}$, Bayer Schering Pharma, Berlin, Germany; $120 \mathrm{~mL}$ ) was injected intravenously using an automatic injector (Stellant, Medrad Europe, Maastricht, The Netherlands) at a rate of $3 \mathrm{~mL} / \mathrm{s}$. The MDCT scanning parameters were as follows: 64 detector rows; beam collimation $64 \times 0.62$; pitch 0.983 ; $\mathrm{kVp} /$ effective $\mathrm{mA} \mathrm{120/300}$; slice thickness $2 \mathrm{~mm}$; slice interval $1 \mathrm{~mm}$. Multiplanar reconstruction images in the coronal plane were obtained at 1-mm intervals (slice thickness $3 \mathrm{~mm}$ ). In accordance with the literature [27] unenhanced, late arterial (40 s after injection), portal venous (70 s) and, if necessary, late phase (130 s) scans were acquired. The total examination time was approximately $10 \mathrm{~min}$.

MDCT images were reviewed independently by two radiologists (F.D.C. and F.G.) who were privy only to tumour location. In the case of discordance, the opinion of a third radiologist (R.N; 26 years of experience of abdominal imaging) was requested.

The depth of infiltration was evaluated in accordance with earlier studies [26]: T1 (mucosal thickening with enhancement); T2 (disruption of a low attenuation stripe); T3 (irregular thickened gastric wall without serosal involvement); $\mathrm{T} 4 \mathrm{a}$ (evidence of the invasion of perilesional adipose tissue); T4b (invasion of adjacent organs). As already stated for MRI, round lymph nodes with a shortaxis diameter of more than $6 \mathrm{~mm}$ and without a normal fatty hilum were considered pathological [26].

EUS protocol and analysis

EUS was performed after instillation of $500 \mathrm{~mL}$ of water into the gastric cavity to improve the transmission of the ultrasound beam, using a linear-array $5-10-\mathrm{MHz}$ ultrasound video endoscope (Hitachi/Pentax EG3870UTK and Hitachi H900 HiVision; Hitachi, Tokyo, Japan). EUS was performed by one experienced endoscopist (P.G.A; 22 years of experience in abdominal EUS) who was privy only to tumour location.

$\mathrm{T}$ staging was evaluated in accordance with the literature [28, 29]: T1 (uneven mucosal surface); T2 (the lesion penetrates the hypoechoic muscularis propria); T3 (the neoplastic tissue reaches the hyperechoic serosal layer); 
T4a (evidence of invasion of the adipose tissue); T4b (invasion of adjacent organs). Similarly to the MRI and MDCT criteria, round and hypoechoic lymph nodes with a short-axis diameter greater than $6 \mathrm{~mm}$ and without a visible hilum were considered pathological [26].

\section{Statistical analysis}

Continuous variables are presented as the mean $( \pm$ the standard deviation), and categorical data are presented as frequencies and percentages. To evaluate the diagnostic performance of the combined use of imaging techniques, multivariate logistic models for both $\mathrm{T}$ stage and $\mathrm{N}$ prediction were fitted on the basis of the imaging modalities considered: tumours were predicted as having serosal invasion and/or nodal involvement when the probability given by $\frac{\exp \left(\alpha_{0}+\sum \alpha_{i} X_{i}\right)}{1+\exp \left(\alpha_{0}+\sum \alpha_{i} X_{i}\right)}$ was greater than 0.5 , where $X_{i}$ are the results of the methods considered and $\alpha_{i}$ represent the odds ratios fitted by the maximum likelihood method. As diagnostic performance measures, sensitivity, specificity, negative predictive value, positive predictive value and accuracy with associated 0.95 confidence intervals (CI) were calculated by means of leave one out cross validation.

Pairwise comparisons of the diagnostic performance measures between the three imaging modalities, or their combined use, for both $\mathrm{T}$ and $\mathrm{N}$, were assessed using the McNemar exact test. Exact $p$ values were computed by means of permutations and considered significant when $<0.05$.

All statistical analyses were performed using R (Foundation for Statistical Computing, Vienna, Austria).

\section{Results}

For the three imaging techniques, all patients $(n=52)$ had visible lesions, and they were treated with radical surgery. According to the Siewert classification [30], seven of 52 $(14 \%)$ were gastro-oesophageal junction lesions (Siewert II and III), which have been proved [31] to be commonly staged as gastric lesions, and 45 of $52(86 \%)$ were proper gastric cancers.

Table 1 shows detailed data of the enrolled population [32].

\section{Multimodality imaging}

As the management of gastric cancer strongly depends on serosal and nodal status, we divided the population into two groups on the basis of the local invasion (T1-3 vs T4a-b) and nodal involvement ( $\mathrm{N} 0$ vs $\mathrm{N}+$ ), and then tested the validity of the three techniques.
Table 1 Characteristics of patients $(n=52)$

\begin{tabular}{|c|c|}
\hline Characteristic & Number \\
\hline \multicolumn{2}{|l|}{ Sex } \\
\hline Male & $33(63 \%)$ \\
\hline Female & $19(37 \%)$ \\
\hline \multicolumn{2}{|l|}{ Site of tumour } \\
\hline Siewert II & $3(6 \%)$ \\
\hline Siewert III & $4(8 \%)$ \\
\hline Stomach & $45(86 \%)$ \\
\hline \multicolumn{2}{|l|}{ Pathological $\mathrm{T}^{\mathrm{a}}$} \\
\hline $\mathrm{T} 1$ & $19(37 \%)$ \\
\hline $\mathrm{T} 2$ & $8(15 \%)$ \\
\hline $\mathrm{T} 3$ & $8(15 \%)$ \\
\hline $\mathrm{T} 4 \mathrm{a}$ & $16(31 \%)$ \\
\hline $\mathrm{T} 4 \mathrm{~b}$ & $1(2 \%)$ \\
\hline \multicolumn{2}{|l|}{ Pathological $\mathrm{N}^{\mathrm{a}}$} \\
\hline No & $25(48 \%)$ \\
\hline N1 (<7 nodes) & $6(12 \%)$ \\
\hline N2 (7-14 nodes) & $7(13 \%)$ \\
\hline N3 (>14 nodes) & $14(27 \%)$ \\
\hline \multicolumn{2}{|l|}{ Histology } \\
\hline Adenocarcinoma & $31(60 \%)$ \\
\hline Signet-ring cell & $21(40 \%)$ \\
\hline \multicolumn{2}{|l|}{ Surgical approach } \\
\hline Ivor-Lewis & $2(4 \%)$ \\
\hline Subtotal gastrectomy & $28(54 \%)$ \\
\hline Total gastrectomy & $22(42 \%)$ \\
\hline \multicolumn{2}{|l|}{ Lauren classification } \\
\hline Intestinal type & $30(58 \%)$ \\
\hline Diffuse type & $17(32 \%)$ \\
\hline Indeterminate & $5(10 \%)$ \\
\hline
\end{tabular}

${ }^{a}$ According to the seventh edition of the TNM classification.

For $\mathrm{T}$ staging (Table 2), the overall accuracy was similar for MRI and MDCT and was lower for EUS, although this did not reach statistical significance $(p=1$ for MRI and MDCT; $p=0.12$ for MRI and EUS; $p=0.21$ for MDCT and EUS). Compared with the other modalities, EUS showed the best sensitivity, significantly higher than MDCT $(p=0.02)$, but, conversely, an inferior specificity $(p=0.003$ and $p=0.0009$ compared with MRI and MDCT).

For $\mathrm{N}$ staging we observed a similar accuracy for MDCT and EUS and slightly lower values for MRI. EUS showed a significantly superior sensitivity in the depiction of pathological nodes when compared with MRI $(p=0.01)$ and MDCT $(p=0.02)$, whereas MDCT showed higher specificity than EUS $(p=0.03)$.

Table 3 shows a substantial overlap in specificity for $\mathrm{T}$ stage in all pairwise comparisons, and the proper 
Table 2 Performance characteristics of techniques for local invasion and metastatic lymph nodes

\begin{tabular}{lllllll}
\hline & MRI & MDCT & EUS & MRI vs MDCT $(p)$ & MRI vs EUS $(p)$ & MDCT vs EUS $(p)$ \\
\hline T stage & & & & & \\
Sensitivity & $76(64-88)$ & $65(52-78)$ & $94(87-100)$ & 0.16 & 0.08 & 0.003 \\
Specificity & $89(80-97)$ & $91(84-99)$ & $60(46-73)$ & 0.32 & 0.12 & 0.0009 \\
Accuracy & $85(75-94)$ & $83(72-93)$ & $71(59-83)$ & 1 & - & - \\
PPV & $76(65-88)$ & $79(67-90)$ & $53(40-67)$ & - & - & - \\
NPV & $88(80-97)$ & $84(74-94)$ & $95(90-100)$ & - & & 0.01 \\
N stage & & & & 0.15 & 0.02 \\
Sensitivity & $69(57-82)$ & $73(61-85)$ & $92(85-99)$ & 0.32 & 0.03 \\
Specificity & $73(61-85)$ & $81(70-91)$ & $58(44-71)$ & 0.16 & - \\
Accuracy & $71(59-83)$ & $77(65-88)$ & $75(63-87)$ & 0.25 & - & - \\
PPV & $72(60-84)$ & $79(68-90)$ & $69(56-81)$ & - & - & - \\
NPV & $70(58-83)$ & $75(63-87)$ & $88(79-97)$ & - & - \\
\hline
\end{tabular}

Data are given as percentages, with 0.95 confidence intervals in parentheses.

$E U S$ endoscopic ultrasonography, $M D C T$ multidetector computed tomography, $M R I$ magnetic resonance imaging, $N P V$ negative predictive value, $P P V$ positive predictive value

Table 3 Multivariate analysis for local invasion and metastatic lymph nodes

Data are given as percentages, with 0.95 confidence intervals in parentheses.

EUS endoscopic

ultrasonography, $M D C T$

multidetector computed

tomography, MRI magnetic

resonance imaging, $N P V$

negative predictive value, $P P V$

positive predictive value

\begin{tabular}{lllll}
\hline & MRI and MDCT & EUS and MDCT & MRI and EUS & MRI, MDCT and EUS \\
\hline T stage & & & & \\
Sensitivity & $76(65-88)$ & $65(51-78)$ & $76(65-88)$ & $76(65-88)$ \\
Specificity & $89(80-97)$ & $91(84-99)$ & $91(84-99)$ & $89(80-97)$ \\
Accuracy & $85(75-94)$ & $83(72-93)$ & $87(77-96$ & $85(75-94)$ \\
PPV & $76(65-88)$ & $79(67-90)$ & $81(71-92$ & $76(65-88)$ \\
NPV & $89(80-97)$ & $84(74-94)$ & $88(80-97)$ & $89(80-97)$ \\
N stage & & & & \\
Sensitivity & $73(61-85)$ & $73(61-85)$ & $92(85-100)$ & $73(61-85)$ \\
Specificity & $81(70-91)$ & $85(75-94)$ & $58(44-71)$ & $62(48-75)$ \\
Accuracy & $77(65-88)$ & $79(68-90)$ & $75(63-87)$ & $67(55-80)$ \\
PPV & $79(68-90)$ & $83(72-93)$ & $69(56-81)$ & $65(53-78)$ \\
NPV & $75(63-87)$ & $76(64-87)$ & $88(79-97)$ & $70(57-82)$ \\
\hline
\end{tabular}

association of EUS and MDCT revealed a slightly lower sensitivity $(65 \%)$. Considering simultaneously all the three imaging modalities, we found the accuracy was similar (Figs. 1, 2). For $\mathrm{N}$ staging, a substantial overlap in accuracy was observed in all pairwise comparisons, whereas the combined association of MRI and EUS revealed the highest sensitivity (92\%) but, conversely, the lowest specificity $(58 \%)$.

For $\mathrm{T}$ staging (Table 4), EUS showed significantly higher sensitivity compared with the association of EUS and MDCT (94\% vs $65 \%, p=0.02$ ). Conversely, the addition or the combined use of MDCT and MRI showed significantly higher specificity than EUS in all comparisons.

For $\mathrm{N}$ staging (Table 5), EUS showed the best sensitivity $(92 \%)$ in all cases $(p=0.02)$ except for the association of EUS and MRI. The association of MRI and EUS showed a significantly higher sensitivity compared with MDCT and MRI (92\% vs $73 \%$ and $69 \% ; p=0.02$ and $p=0.01$, respectively) and the same sensitivity as EUS $(92 \% ; p=1)$. Conversely, MDCT showed a higher specificity than both the association of EUS and MRI and all the techniques clustered together $(81 \%$ vs $58 \%$ and $62 \%, p=0.03$ and $p=0.05$, respectively). The association of MDCT and MRI showed higher specificity than EUS ( $81 \%$ vs $58 \%, p=0.03)$.

\section{Discussion}

Since there is as yet no consensus on the optimum modality for the staging of gastric cancer, our prospective study was 

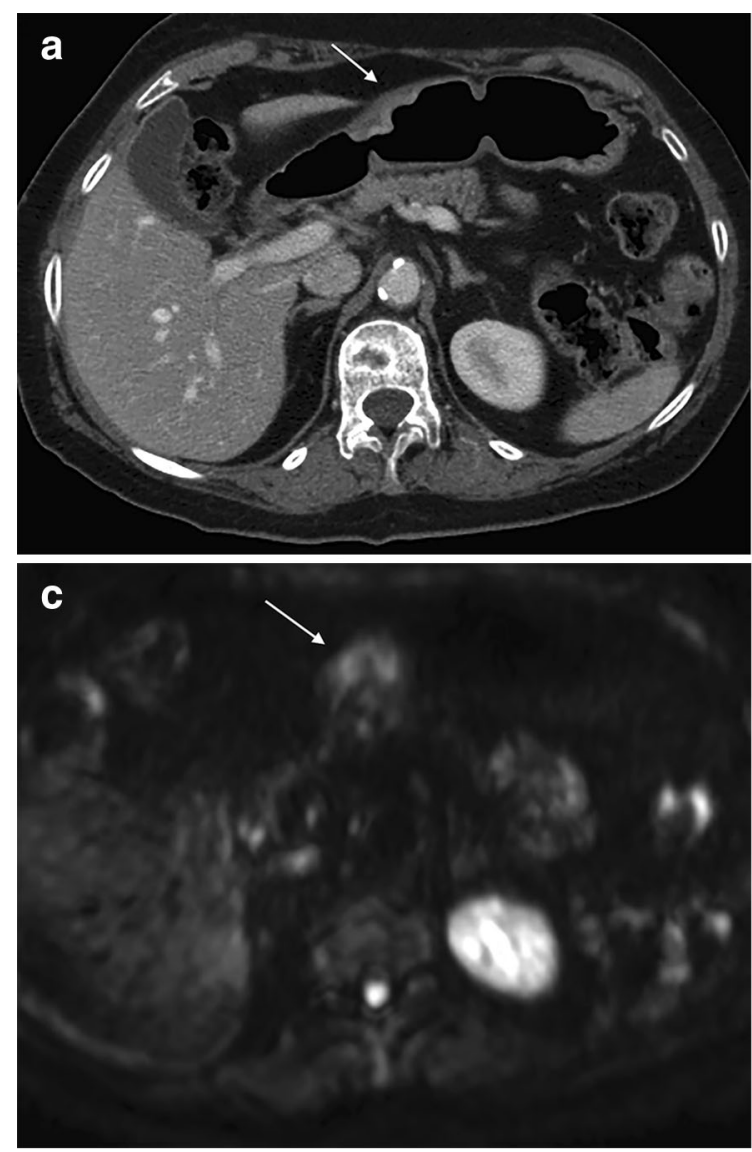

Fig. 1 Lesion involving the antrum in a 77-year-old woman. Axial postcontrast computed tomography (a), axial T2 (b) and diffusionweighted imaging (c) scans and endoscopic ultrasonography (d) shot. In a the arrow indicates mucosal and submucosal thickening with focal enhancement. In $\mathbf{b}$ the arrow shows focal thickening of the inner layers of the gastric wall along with slight invasion of the muscularis

designed to investigate the preoperative diagnostic performance of MRI, MDCT and EUS.

This is the first in vivo study where MRI, MDCT and EUS were performed in the same population of patients in whom gastric cancer had been diagnosed. Moreover, we included only patients treated directly with surgery to remove any confounding biological changes resulting from neoadjuvant therapy; as such, each diagnostic modality faced the same case load (e.g. peritumoral inflammation, serosal and nodal involvement).

MRI accuracy for $\mathrm{T}$ staging was similar to that of MDCT, whereas a lower value was found for EUS. The literature yields accuracy values ranging from 69 to $89 \%$ for MDCT and from 73.5 to $87.5 \%$ for MRI along with an accuracy of $76.7 \%$ for EUS and $78.2 \%$ for MDCT, respectively, without significant differences $[16,33]$. In our study EUS showed the highest sensitivity, underlining the
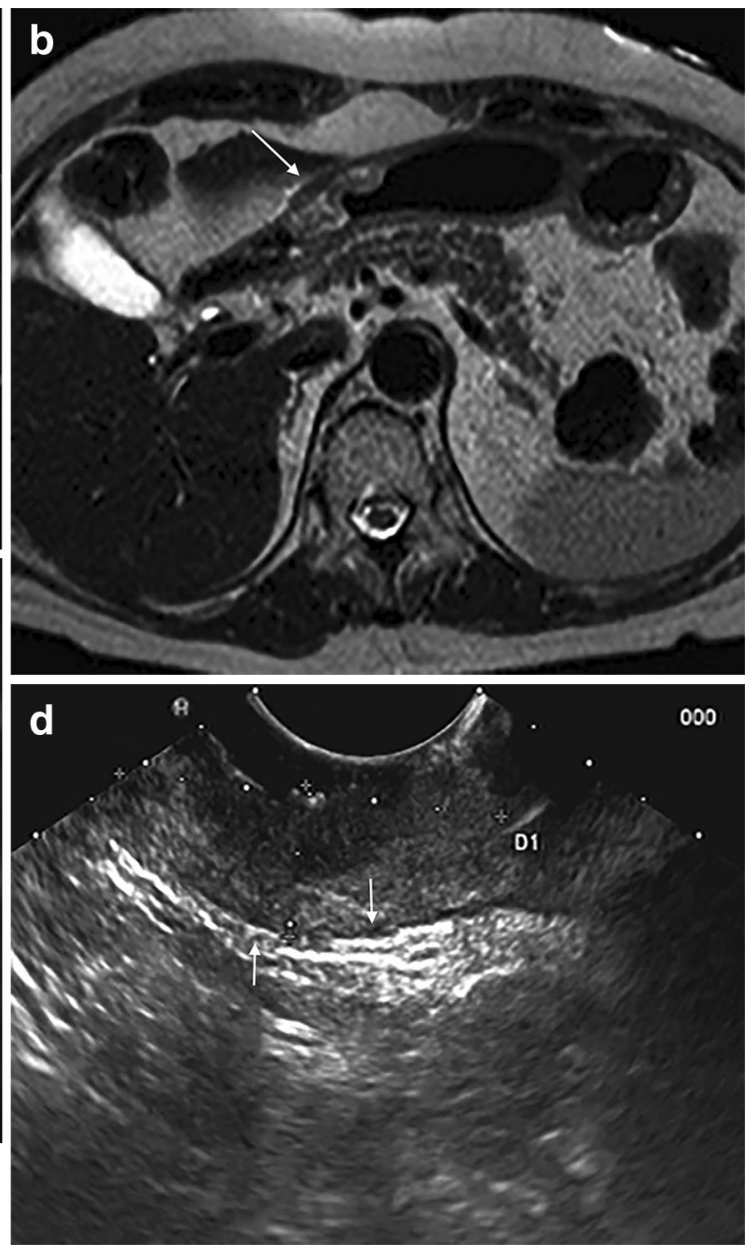

propria, and in $\mathbf{c}$ the high signal intensity of the lesion is recognizable. In $\mathbf{d}$ the arrows indicate the entirety of the hyperechoic serosal layer as the lesion penetrates only the hypoechoic muscularis propria above. All three imaging modalities properly staged this lesion as "non-invasive" (T2), as proved by histopathological evaluation. The apparent diffusion coefficient for the lesion was $1.6 \times 10^{-3} \mathrm{~mm}^{2} / \mathrm{s}$

primary role of this technique in the detection of locally advanced tumours and, thus, its capability to correctly recommend neoadjuvant therapy instead of immediate surgical resection.

Conversely, MRI and MDCT resulted in significant higher specificity, showing the capability of both techniques to better identify tumours without serosal invasion (whose optimal treatment is immediate surgical resection). Of note, this aspect is very important to avoid risks correlated with non-recommended neoadjuvant therapy (e.g. infections due to bone marrow toxicity).

As stated before, the detection of serosal involvement is an important factor as it defines the best curative approach (neoadjuvant therapy prior to surgery). In terms of diagnostic imaging, the most important aspect to stage serosal invasion is represented by spatial resolution and soft tissue contrast. It is well known that MDCT does not offer the 

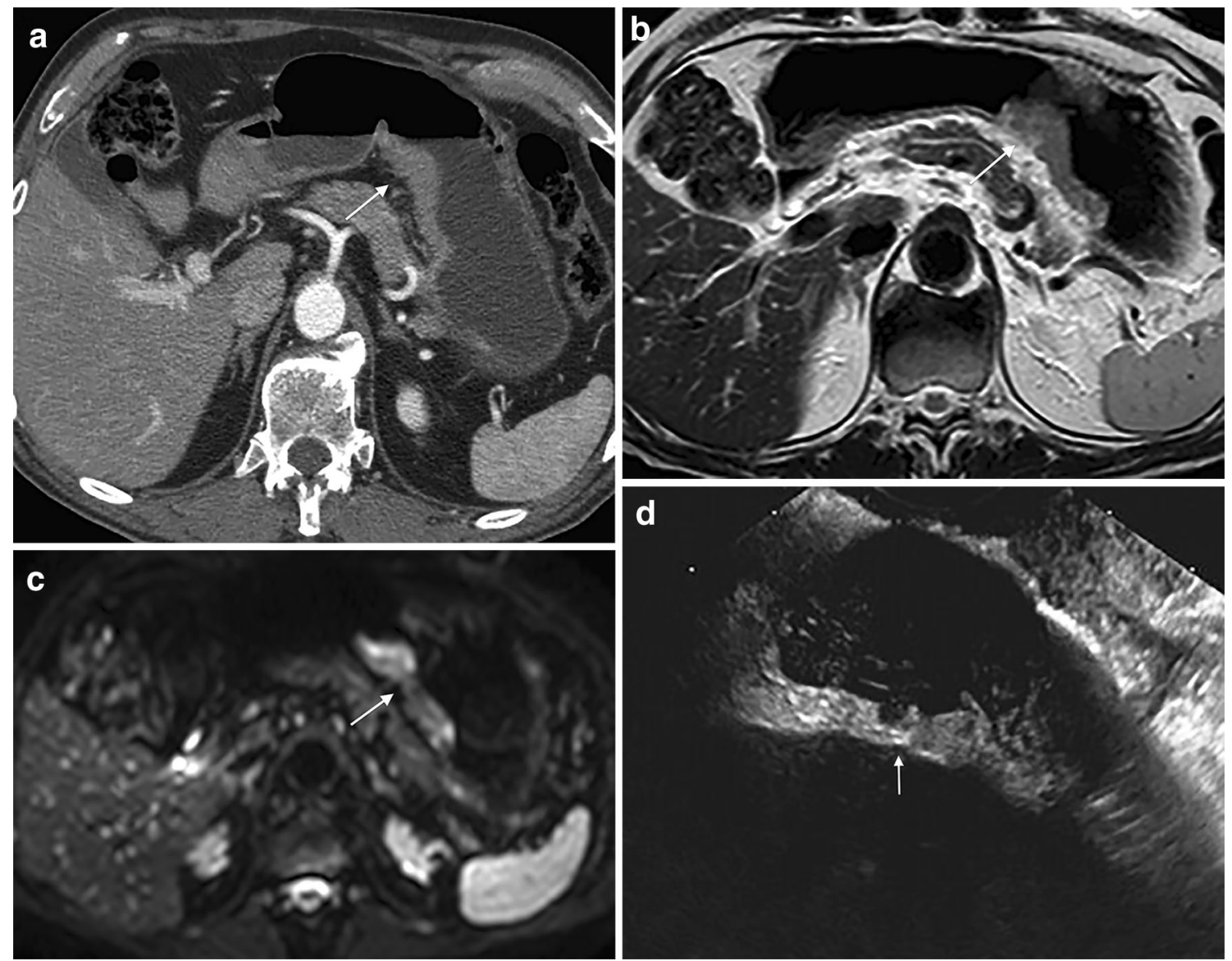

Fig. 2 Lesion involving the lesser gastric curvature in a 71-year-old man. Axial postcontrast computed tomography (a), axial T2 (b) and diffusion-weighted imaging (c) scans and endoscopic ultrasonography (d) shot. In a the arrow indicates the irregular outer borders of the thickened gastric wall with evidence of subserosal and perigastric fat invasion. In $\mathbf{b}$ the arrow shows a thickened gastric wall associated

with transmural extension into perigastric fat along with high signal intensity on diffusion-weighted imaging (c). In $\mathbf{d}$ the invasion of the serosa and transmural extension into perigastric fat are recognizable. All three imaging modalities properly staged this lesion as "locally advanced" (T4a), as proved by histopathological evaluation. The apparent diffusion coefficient for the lesion was $1.2 \times 10^{-3} \mathrm{~mm}^{2} / \mathrm{s}$

Table 4 Multivariate analysis between techniques for local invasion

\begin{tabular}{|c|c|c|c|c|}
\hline \multicolumn{5}{|l|}{ Sensitivity } \\
\hline & MDCT and EUS (65\%) & MDCT and MRI (76 \%) & EUS and MRI (76 \%) & MDCT, EUS and MRI (76 \%) \\
\hline $\operatorname{MDCT}(65 \%)$ & 1 & 0.15 & 0.15 & 0.15 \\
\hline MRI (76 \%) & 0.15 & 1 & 1 & 1 \\
\hline EUS (94\%) & 0.02 & 0.08 & 0.08 & 0.08 \\
\hline \multicolumn{5}{|l|}{ Specificity } \\
\hline & MDCT and EUS (91\%) & MDCT and MRI (89 \%) & EUS and MRI (91\%) & MDCT, EUS and MRI (89 \%) \\
\hline MDCT $(91 \%)$ & 1 & 0.31 & 1 & 0.31 \\
\hline MRI (89\%) & 0.31 & 1 & 0.31 & 1 \\
\hline EUS $(60 \%)$ & 0.0009 & 0.003 & 0.0009 & 0.003 \\
\hline
\end{tabular}

Data are given as $p$ values.

EUS endoscopic ultrasonography, MDCT multi-detector computed tomography, MRI magnetic resonance imaging 
Table 5 Multivariate analysis between techniques for nodal involvement

\begin{tabular}{|c|c|c|c|c|}
\hline \multicolumn{5}{|l|}{ Sensitivity } \\
\hline & MDCT and EUS (73 \%) & MDCT and MRI (73\%) & EUS and MRI (92\%) & MDCT, EUS and MRI (73 \%) \\
\hline MDCT (73\%) & 1 & 1 & 0.02 & 1 \\
\hline MRI (69 \%) & 0.31 & 0.31 & 0.01 & 0.31 \\
\hline EUS (92\%) & 0.02 & 0.02 & 1 & 0.02 \\
\hline \multicolumn{5}{|l|}{ Specificity } \\
\hline & MDCT and EUS (85 \%) & MDCT and MRI (81\%) & EUS and MRI (58 \%) & MDCT, EUS and MRI (62 \%) \\
\hline MDCT (81\%) & 0.31 & 1 & 0.03 & 0.05 \\
\hline MRI (73 \%) & 0.08 & 0.15 & 0.15 & 0.31 \\
\hline EUS (58 \%) & 0.08 & 0.03 & 1 & 0.31 \\
\hline
\end{tabular}

Data are given as $p$ values.

EUS endoscopic ultrasonography, $M D C T$ multidetector computed tomography, MRI magnetic resonance imaging

same soft tissue contrast as EUS and MRI [8, 11, 27], and this aspect is in line with our results, showing that EUS and MRI have considerably higher sensitivity.

In the multivariate analysis for $\mathrm{T}$ staging, we observed a significantly higher EUS sensitivity when EUS was compared with MDCT; moreover, MRI improved MDCT sensitivity when both techniques were considered together, but without a significant difference. The explanation for this result is attributable to the high resolution and excellent soft tissue contrast typical of MRI (especially when compared with MDCT [34]) as the differentiation between $\mathrm{T} 3$ and T4a on MDCT is very difficult owing to the impossibility to visualize the serosa properly.

However, as already stated, MRI has to cope with motion artefacts (e.g. peristalsis and patient movement) and these aspects, unfortunately, represent a great limitation in the staging of gastric cancer, with the risk of leading to erroneous results; moreover, MRI is not able to detect distant metastases, but MDCT can.

Therefore, our results confirm the leading role of EUS in the detection of serosal involvement and support the evidence that MDCT is a well-established method to identify metastases, suggesting that, at present, the use of MRI is not justified in the staging of gastric cancer.

For N stage, EUS showed the highest sensitivity (i.e. the capability to correctly identify pathological nodes) and MDCT showed the highest specificity. As there is notable interobserver variation in pathological $\mathrm{N}$ staging, lymph nodes should be measured using a reproducible method (i.e. the short-axis diameter should be measured perpendicular to the longest one).

As a result, since there is still no worldwide consensus for diagnosing metastatic lymph nodes (e.g. pathological nodal size ranging from more than $5 \mathrm{~mm}$ to more than
$1 \mathrm{~cm})[26,35]$ and each technique uses different criteria for assessing nodal involvement, we deliberately chose a common cut-off of $6 \mathrm{~mm}$ on the short axis (which is a widespread criterion applied to distinguish metastatic perigastric lymph nodes) [26] for all the three techniques in order to ensure the reproducibility of our results.

The overall accuracies of MRI, MDCT and EUS for N staging were not completely satisfactory in our study and this may be explained bearing in mind that pathological nodes are not always enlarged and swollen nodes are not always malignant but can be simply inflammatory $[25,26$, 29].

As a consequence, it is clear that these aspects represent some important limitations in the management of gastric cancer and point out why nodal assessment is still extremely difficult. In our opinion, further studies should aim at finding homogeneous and well-established diagnostic criteria for $\mathrm{N}$ staging, applicable to different imaging modalities. However, despite some limitations (e.g. the low sensitivity), MDCT still remains the most commonly used imaging modality for $\mathrm{N}$ staging in gastric cancer by virtue of its panoramic field of view.

We acknowledge that our study has some limitations. Firstly, a great number of patients $(n=62)$ were discarded owing to our restrictive exclusion criteria, resulting in a small but, at the same time, homogeneous population that was treated directly with surgery. Therefore, one of our aims is to include even patients suitable for endoscopic resection or candidates for neoadjuvant therapy.

Moreover, a clear weakness of our prospective study is that the cohort was composed, at the final histological evaluation, of a great number of patients with T1-T3 tumours ( 35 patients) and a smaller number of patients with T4a-b tumours (17 patients); as stated before, this 
discrepancy is due to the lack of adequate preoperative imaging techniques and, once again, another aim of our group will be to enrol a higher number of locally advanced tumours that are not addressed with neoadjuvant therapy but are treated directly with surgery.

Finally, we deem that some differences concerning the diagnostic protocol (e.g. not using scopolamine butylbromide for EUS) may have led to a decrease of EUS accuracy.

\section{Conclusion}

Our prospective study has provided initial evidence that, at present, the preoperative locoregional staging of gastric cancer does not benefit from the potential use of MRI, despite its high contrast resolution and characteristic soft tissue contrast. Since our results showed no significant differences between MDCT and MRI for T and N staging, we can assume that EUS and MDCT or EUS and MRI should be done for the preoperative staging of gastric cancer. Therefore, it is realistic to confirm the importance of a global consideration of EUS and MDCT results for preoperative $\mathrm{T}$ and $\mathrm{N}$ staging in gastric cancer, as both techniques have characteristic advantages in terms of sensitivity and specificity. Hence, further studies are needed, and it is hoped that other high-volume centres may evaluate the introduction of MRI in the pathway of preoperative locoregional staging of gastric cancer.

Acknowledgments We thank the patients and their families as well as all the health care assistants (nurses, radiographers) who contributed greatly to the realization of this work.

\section{References}

1. Jemal A, Bray F, Center MM, Ferlay J, Ward E, Forman D. Global cancer statistics. CA Cancer J Clin. 2011;61:69-90.

2. Hohenberger P, Gretschel S. Gastric cancer. Lancet. 2003;362: 305-15.

3. Cunningham D, Allum WH, Stenning SP, Thompson JN, Van de Velde CJ, Nicolson M, et al. Perioperative chemotherapy versus surgery alone for resectable gastroesophageal cancer. N Engl J Med. 2006;355:11-20.

4. Ajani JA, Winter K, Okawara GS, Donohoue JH, Pisters PW, Crane $\mathrm{CH}$, et al. Phase II trial of preoperative chemoradiation in patients with localized gastric adenocarcinoma (RTOG 9904): quality of combined modality therapy and pathologic response. J Clin Oncol. 2006;24:3953-8.

5. Ziegler K, Sanft C, Zimmer T, Zeitz M, Felsenberg D, Stein H, et al. Comparison of computed tomography, endosonography, and intraoperative assessment in TN staging of gastric carcinoma. Gut. 1993;34:604-10.

6. Ahn HS, Lee HJ, Yoo MW. Diagnostic accuracy of T and N stages with endoscopy, stomach protocol CT, and endoscopic ultrasonography in early gastric cancer. J Surg Oncol. 2009; 99(1):20-7.
7. Tsuzuki T, Okada H, Kawahara Y, Nasu J, Takenaka R, Inoue M, et al. Usefulness and problems of endoscopic ultrasonography in prediction of the depth of tumor invasion in early gastric cancer. Acta Med Okayama. 2011;65:105-12.

8. Kwee RM, Kwee TC. Imaging in local staging of gastric cancer: a systematic review. J Clin Oncol. 2007;25:2107-16.

9. Habermann CR, Weiss F, Riecken R, Honarpisheh H, Bohnacker $\mathrm{S}$, Staedtler C, et al. Preoperative staging of gastric adenocarcinoma: comparison of helical CT and endoscopic US. Radiology. 2004;230:465-71.

10. Hwang SW, Lee DH, Lee SH, Park YS, Hwang JH, Kim JW, et al. Preoperative staging of gastric cancer by endoscopic ultrasonography and multidetector-row computed tomography. J Gastroenterol Hepatol. 2010;25:512-8.

11. Chen CY, Wu DC, Kang WY, Hsu JS. Staging of gastric cancer with 16-channel MDCT. Abdom Imaging. 2006;31:514-20.

12. Kim AY, Kim HJ, Ha HK. Gastric cancer by multidetector row CT: preoperative staging. Abdom Imaging. 2005;30:465-72.

13. Kumano S, Muratami T, Kim T, Hori M, Iannacone R, Nakata S, et al. T staging of gastric cancer: role of multi-detector row CT. Radiology. 2005;237:961-6.

14. Chen CY, Hsu JS, Wu DC, Kang WY, Hsieh JS, Jaw TS, et al. Gastric cancer preoperative local staging with 3D multi-detector row CT-correlation with surgical and histopathologic results. Radiology. 2007;242:472-82.

15. Yang DM, Kim HC, Jin W, Ryu CW, Kang JH, Park CH, et al. 64 multidetector-row computed tomography for preoperative evaluation of gastric cancer histological correlation. J Comput Assist Tomogr. 2007;31:98-103.

16. Anzidei M, Napoli A, Zaccagna F, Di Paolo P, Zini C, Cavallo Marincola B. Diagnostic performance of 64-MDCT and 1.5-T MRI with high resolution sequences in the $\mathrm{T}$ staging for gastric cancer: a comparative analysis with histopathology. Radiol Med. 2009;114:1065-79.

17. Giganti F, De Cobelli F, Canevari C, Orsenigo E, Gallivanone F, Esposito A, et al. Response to chemotherapy in gastric adenocarcinoma with diffusion-weighted MRI and ${ }^{18}$ F-FDG-PET/CT: correlation of apparent diffusion coefficient and partial volume corrected standardized uptake value with histological tumor regression grade. J Magn Reson Imaging. 2013;40 (5):1147-57. doi:10.1002/jmri.2013.24464.

18. Kang BC, Kim JH, Kim KW, Lee DY, Baek SY, Lee SW, et al. Value of the dynamic and delayed MR sequence with Gd-DTPA in the $\mathrm{T}$ staging of stomach cancer: correlation with histopathology. Abdom Imaging. 2000;25:14-24.

19. Sohn KM, Lee JM, Lee SY, Ahn BY, Park SM, Kim KM. Comparing MR imaging and CT in the staging of gastric carcinoma. AJR Am J Roentgenol. 2000;174:1551-7.

20. Kim AY, Han JK, Seong CK, Kim TK, Choi B. MRI in staging advanced gastric cancer: is it useful compared with spiral CT? J Comput Assist Tomogr. 2000;24(3):389-94.

21. Heye T, Kuntz C, Dux M, Encke J, Palmowski M, Autschbach F, et al. CT and endoscopic ultrasound in comparison to endoluminal MRI-preliminary results in staging gastric carcinoma. Eur J Radiol. 2009;70(2):336-41.

22. Peng CW, Wang LW, Zeng WJ, Yang XJ, Li Y. Evaluation of the staging systems for gastric cancer. J Surg Oncol. 2013;108(2): 93-105.

23. De Cobelli F, Giganti F, Orsenigo E, Cellina M, Esposito A, Agostini G, et al. Apparent diffusion coefficient modifications in assessing gastro-oesophageal cancer response to neoadjuvant treatment: comparison with tumour regression grade at histology. Eur Radiol. 2013;23(8):2165-74.

24. Palmowski M, Grenacher L, Kuntz C, Heye T, Dux M. Magnetic resonance imaging for local staging of gastric carcinoma: results of an in vitro study. J Comput Assist Tomogr. 2006;30:896-902. 
25. Kim IY, Kim SW, Shin HC, Lee MS, Jeong DJ, Kim CJ, et al. MRI of gastric carcinoma: results of $\mathrm{T}$ and $\mathrm{N}$-staging in an in vitro study. World J Gastroenterol. 2009;15(32):3992-8.

26. Lee MH, Choi D, Park MJ, Lee MW. Gastric cancer: imaging and staging with MDCT based on the 7th AJCC guidelines. Abdom Imaging. 2012;37(4):531-40.

27. Chen CY, Hsu HS, Wu DC, Kang WY, Hsieh JS, Jaw TS. Gastric cancer: preoperative local staging with $3 \mathrm{D}$ multidetector row CT-correlation with surgical and histopatological results. Radiology. 2007;242(2):472-82.

28. Arocena MG, Barturen A, Bujanda L, Casado O, Ramirez MM, Oleagoitia JM, et al. MRI and endoscopic ultrasonography in the staging of gastric cancer. Rev Esp Enferm Dig. 2006;98(8): 582-90.

29. Cho JW. The role of endoscopic ultrasonography in T staging: early gastric cancer and esophageal cancer. Clin Endosc. 2013; 46(3):239-42.

30. Siewert JR, Stein HJ. Classification of adenocarcinoma of the oesophagogastric junction. Br J Surg. 1998;85:1457-9.
31. Hasegawa S, Yoshikawa T, Aoyama T, Hayashi T, Yamada T, Tsuchida K, et al. Esophagus or stomach? The seventh TNM classification for Siewert type II/III junctional adenocarcinoma. Ann Surg Oncol. 2013;20(3):773-9.

32. Hamilton SR, Aaltonen LA, editors. World Health Organization classification of tumours. Vol. 3, Pathology \& genetics. Tumours of the digestive system. 3rd ed. Lyon: IARC Press; 2000.

33. Feng XY, Wang W, Luo GY, Wu J, Zhou ZW, Li W, et al. Comparison of endoscopic ultrasonography and multislice spiral computed tomography for the preoperative staging of gastric cancer-results of a single institution study of 610 chinese patients. PLoS One. 2013;8(11):e78846.

34. Maccioni F, Marcelli G, Al Ansari N, Zippi M, De Marco V, Kagarmanova A. Preoperative T and N staging of gastric cancer: magnetic resonance imaging (MRI) versus multi detector computed tomography (MDCT). Clin Ter. 2010;161(2):57-62.

35. Choi JI, Joo I, Lee JM. State-of-the-art preoperative staging of gastric cancer by MDCT and magnetic resonance imaging. World J Gastroenterol. 2014;20(16):4546-57. 